\title{
Riesz potential versus fractional Laplacian
}

\author{
Manuel D Ortigueira, Taous-Meriem Laleg-Kirati and J A Tenreiro Machado
}

\begin{abstract}
This paper starts by introducing the Grünwald-Letnikov derivative, the Riesz potential and the problem of generalizing the Laplacian. Based on these ideas, the generalizations of the Laplacian for 1D and 2D cases are studied. It is presented as a fractional version of the Cauchy-Riemann conditions and, finally, it is discussed with the $n$-dimensional Laplacian.
\end{abstract}

Keywords: nonlinear dynamics

\section{Introduction}

The Laplacian is an important operator in physics and engineering. However, the standard definition does not cope with domains that are neither homogeneous, nor isotropic. Nowadays, researchers in applied sciences are interested in finding a definition of fractional Laplacian suitable for studying both isotropic and anisotropic media. In recent studies the fractional Laplacian was implemented by means of the inverse of the Riesz potential [1,2]. However, it is not straightforward that this is the single option when thinking about several distinct types of applications. In fact, in $n \mathrm{D}$ applications the inverse of the Riesz potential cannot be expressed as a sum of partial derivatives and is only defined using the Fourier transform inverse of an operator. An alternative option is to follow the vectorial calculus that allows finding a Laplacian as the sum of partial derivatives [3-5]. In an attempt to gain a deeper insight, in this paper we develop a heuristic analysis of the Riesz potential, in the perspective of what we are expecting in a Laplacian. As a collateral result, a possible generalization of the Cauchy-Riemann conditions is obtained.

Bearing these ideas in mind the paper is organized as follows. In section 2 we introduce the Grünwald-Letnikov derivative and the Riesz potential. In section 3 we formulate the problem of generalising the Laplacian presenting two alternatives. It discusses the adoption of the theories of powers of operators [6], and of the generalized functions with Schwartz type test spaces [7]. In section 4 we revisit the generalization of the Laplacian for 1D and 2D cases. Starting from the current integer order, the Laplacian are discussed and conjectures about its generalization are made by analyzing the 1D and 2D cases. Furthermore, a fractional version of the Cauchy-Riemann conditions is presented and the $n \mathrm{D}$ case considered. Finally, in section 5 we outline the main conclusions. 


\section{The Grünwald-Letnikov and Riesz derivatives}

We define the forward and backward Grünwald-Letnikov (GL) derivatives [8-11] by the equations:

$$
\begin{aligned}
D_{f}^{\alpha} f(t) & =\lim _{|h| \rightarrow 0} \frac{\sum_{j=0}^{\infty}(-1)^{j}\left(\begin{array}{l}
\alpha \\
j
\end{array}\right) f(t-j h)}{|h|^{\alpha}}, \\
D_{b}^{\alpha} f(t) & =\mathrm{e}^{-\mathrm{i} \alpha \pi} \lim _{|h| \rightarrow 0} \frac{\sum_{j=0}^{\infty}(-1)^{j}\left(\begin{array}{c}
\alpha \\
j
\end{array}\right) f(t+j|h|)}{|h|^{\alpha}},
\end{aligned}
$$

The two expressions are easily extended to define partial derivatives. Here, without lack of generality, we will adopt the forward derivatives.

The so-called Riesz potential operators were introduced by Marcel Riesz [12] looking for generalizations of the Riemann-Liouville (RL) operator for dealing with the wave equation. The concept was built on the theory for the 1D RL fractional operators and Liouville (see [7, 8]).

Many authors used the 1D Riesz operator on the spatial variable that, in fact, may be considered as variants of the corresponding operators introduced by Riesz [12] and Feller [13].

Riesz introduced three $n \mathrm{D}$ integral potential operators to calculate explicitly the potential for hyperbolic, elliptic and parabolic Cauchy problems. In our study the most interesting is the weak singular integral operator $I^{a}$ defined by Riesz in [12]

$$
\left(\mathbb{I}_{R}^{\alpha} f\right)(\bar{x}):=\frac{1}{\gamma_{n}(\alpha)} \int_{\mathbb{R}^{n}} f(\bar{y})|\bar{x}-\bar{y}|^{\alpha-n} \mathrm{~d} \bar{y},
$$

where $0<\alpha<n$ with $\alpha-n \neq 2 k, k \in \mathbb{N}_{0}$, and $f$ are suitable functions. For example, locally integrable functions on $\mathbb{R}^{n}$, or with sufficiently rapid decaying at infinity, as when $f \in L p\left(\mathbb{R}^{n}\right)$ with $1 \leqslant p<\frac{n}{\alpha}$. The normalized constant $\gamma_{n}(\alpha)$ is given by

$$
\gamma_{n}(\alpha)=\frac{\pi^{\frac{n}{2}} 2^{\alpha} \Gamma\left(\frac{\alpha}{2}\right)}{\Gamma\left(\frac{n-\alpha}{2}\right)}=2 \frac{\pi^{\frac{n+1}{2}} \Gamma(\alpha)}{\Gamma\left(\frac{n-\alpha}{2}\right) \Gamma\left(\frac{1+\alpha}{2}\right)}
$$

If $a-n=2 k, k \in \mathrm{N}_{0}$, the Riesz operator becomes a logarithmic operator [7,12].

In the works of Riesz, the $n \mathrm{D}$ potential operators preserved some of the main properties of the RL fractional integral operators. For suitable functions and $\bar{x} \in \mathrm{R}^{n}$, Riesz proved that

$$
\mathbb{I}_{R}^{\alpha}\left(\mathbb{I}_{R}^{\beta} f\right)(\bar{x})=\left(\mathbb{I}_{R}^{\alpha+\beta} f\right)(\bar{x})
$$


where $0 \leqslant \alpha, \beta, \alpha+\beta \leqslant n$, and

$$
\Delta\left(\mathbb{I}_{R}^{\alpha+2} f\right)(\bar{x})=-\left(\mathbb{I}_{R}^{\alpha} f\right)(\bar{x}),
$$

where $\Delta$ is the classical Laplacian and $0 \leqslant \alpha, \alpha+2 \leqslant n$.

\section{The Laplacian}

This section formulates the problem of generalising the Laplacian. Section 3.1 presents the main definitions. Section 3.2 revisits the Riesz Laplacian. Section 3.3 discusses two alternative strategies for generalization.

\subsection{Definition}

The Laplacian is a useful operator that in the $n \mathrm{D}$ case can be formulated as

$$
\Delta f(\bar{x})=\sum_{j=1}^{n} \frac{\partial^{2} f(\bar{x})}{\partial x_{j}^{2}}
$$

where $\bar{x}=\left(x_{1}, x_{2}, \ldots, x_{n}\right)$. This operator is fundamental in important applications in physics such as in electromagnetism (e.g. the wave equation).

With the Fourier transform we can rewrite the above equation as

$$
\mathcal{F}(-\Delta f)(\bar{k})=|\bar{k}|^{2} \hat{f}(\bar{k})
$$

with

$$
|\bar{k}|^{2}=\sum_{j=1}^{n} k_{j}^{2}
$$

\subsection{The Riesz Laplacian}

In his works, Riesz implicitly suggested several possible definitions of fractional Laplacian [12]. Such expressions are suitable to be adopted in applications involving models with fractional operators on spacial variables. Riesz did not present the explicit realization of such operators, but he deduced expressions like (6) that suggest that the potential, as defined in (3) for positive $a$, could implement the inverse of the integer order Laplacian. It is possible to prove [14] that, for suitable functions $f(\bar{x}), \bar{x}_{\in} \mathrm{R}^{n}$, the aforementioned definitions of fractional Laplacian operator implied by Riesz can be given as follow, for any real $m-\frac{1}{2}<\alpha \leqslant m(m \in \mathbb{N})$ :

$$
\begin{aligned}
& \left((-\Delta)^{\alpha} f\right)(\bar{x}):=(-\Delta)^{m}\left(\mathbb{I}^{2 m-2 \alpha} f\right)(\bar{x}), \quad n \geqslant 1 \\
& \left(\Delta^{\alpha} f\right)(\bar{x}):=-(-\Delta)^{m}\left(\mathbb{I}^{2 m-2 \alpha} f\right)(\bar{x}), \quad n \geqslant 1
\end{aligned}
$$

These two differential operators are the inverse on the left of the potential Riesz integral, given by (3), apart a constant. However, the current applications of the fractional Laplacian have not been based on such relations, as we will see in the sequel. 


\subsection{Strategies for generalizations}

The importance of the Laplacian motivated its generalization towards the fractional case. Two different cases are possible:

- To compute a power of the integer order Laplacian operator

- To fractionalise each (integer order) partial derivative.

These two strategies lead to different solutions. The first has been privileged since there is an expedite way of implementation by means of the Riesz potential. In the positive integer order case it is nothing other than the repeated application of the above operator. If $a<0$ the inverse Fourier transform of $|\bar{k}|{ }^{a}$ leads immediately to the Riesz potential.

In the literature several authors have found the expression for a Riesz Laplacian operator following two ways:

- With the theory of powers of operators [6]

- Using the theory of generalized functions spaces with Schwartz type test spaces [7].

In both cases researchers look for an operator satisfying the following property:

$$
\mathcal{F}\left((-\Delta)^{\alpha} f\right)(\bar{k})=|k|^{\alpha} \mathcal{F}(f)(\bar{k})
$$

with $1<\alpha \leqslant 2$ and

$$
|\bar{k}|^{\alpha}=\left[\sum_{j=1}^{n} k_{j}^{2}\right]^{\frac{\alpha}{2}}
$$

This operator can be implemented by [7]

$$
(-\Delta)^{\alpha} f(\bar{x}):=\frac{1}{\gamma_{n}(\alpha)} \int_{\mathbb{R}^{n}} \frac{\left(\Delta_{y}^{l} f\right)(\bar{x})}{|\bar{x}-\bar{y}|^{2 \alpha+n}} d \bar{y}
$$

where $\left(\Delta^{l} f_{y}\right)(x)$ is the $l$-th difference of $f(x)$. For $l>2 a$ the above integral is absolutely convergent. Frequently $a<1$ and $l$ is taken to be 1 . [1,2]

$$
(-\Delta)^{\alpha} f(\bar{x}):=\frac{1}{\gamma_{n}(\alpha)} \int_{\mathbb{R}^{n}} \frac{f(\bar{y})-f(\bar{x})}{|\bar{x}-\bar{y}|^{2 \alpha+n}} d \bar{y},
$$

This operator has been used to implement the fractional Laplacian [1,2]. However, it does not implement expression (12) exactly [7].

Alternatively, we can start from (7) and we are led to a formulation of the type [15]:

$$
\Delta^{\alpha} f(\bar{x})=\sum_{j=1}^{n} \frac{\partial^{2 \alpha} f(\bar{x})}{\partial x_{j}^{2 \alpha}}
$$

This approach would be interesting because it recovers some properties of the classic vectorial calculus, namely the relationship with the gradient and divergence. With $a=1$ we recover (7). 
In this discussion we add another fact. Let us find the bi-Laplacian $\Delta^{2}=\Delta \Delta$. It is possible to demonstrate that it is given by:

$$
\Delta^{2}=\sum_{j=1}^{n} \frac{\partial^{4} f(\bar{x})}{\partial x_{j}^{4}}+2 \sum_{l=1}^{n} \sum_{j=1, j \neq l}^{n} \frac{\partial^{4} f(\bar{x})}{\partial x_{l}^{2} \partial x_{j}^{2}}
$$

where we verify the emergence of cross derivative terms.

The corresponding Fourier transform is

$$
\mathcal{F}\left(\Delta^{2} f\right)(\bar{k})=|\bar{k}|^{4} \hat{f}(\bar{k})=\left(\sum_{j=1}^{n} k_{j}^{2}\right)^{2} \hat{f}(\bar{k})
$$

in agreement with the Riesz potential approach.

\section{Towards the fractional Laplacian}

This section develops a deeper insight into the available options in the generalization. Section 4.1 starts by addressing the 1D Laplacian. Section 4.2 formulates the 2D Laplacian and the fractional Cauchy-Riemann conditions. Section 4.3 obtains the $n \mathrm{D}$ Laplacian. Section 4.4 discusses some of outcomes that emerge with the new concepts.

\subsection{The 1D Laplacian}

Let $f(x)$ be a function with real variable. We define the GL centred (two-sided) fractional derivative [16-18]:

$$
D_{c}^{\alpha} f(x)=\lim _{h \rightarrow 0} \frac{\sum_{j=-\infty}^{+\infty}(-1)^{j} \frac{\Gamma(\alpha+1)}{\Gamma\left(\frac{\alpha}{2}-j+1\right) \Gamma\left(\frac{\alpha}{2}+j+1\right)} f(x-j h)}{h^{\alpha}}
$$

for $\alpha>-1$. Its Fourier transform is given by:

$$
\mathcal{F}\left[D_{c 1}^{\alpha} f(x)\right](k)=|k|^{\alpha} \hat{f}(k)
$$


As shown in $[17,19]$, this expression reveals that the derivative is equivalent to the $1 \mathrm{D}$ Riesz potential. For $a=1$ we obtain the centred order one derivative that we can consider the half order Laplacian. The result cannot be obtained from the Riesz integral since it leads to a singular integral. For $a=2$ we obtain the order two centred derivative that we can consider the 1D Laplacian (aside a minus sign).

We conclude that in the 1D case this derivative and the Riesz potential give the same Laplacian that differs from the classical one by a minus sign.

\subsection{The 2D Laplacian and the fractional Cauchy-Riemann conditions}

It is very common to treat the $2 \mathrm{D}$ case using complex variable theory. In particular the socalled harmonic functions solution of the Laplacian equations are obtained with the help of the Cauchy-Riemann conditions. These establish relations among the partial derivatives of the real and imaginary parts of a given complex function

$$
\text { where } \mathrm{i}=\sqrt{-1} \text {. }
$$

$$
f(z)=u(x, y)+\mathrm{i} v(x, y), \quad z=x+\mathrm{i} y
$$

In terms of the complex plane and given (3) they correspond to the computation of the derivative on the real and imaginary axis. We now generalize the classical results for the fractional case. We define fractional derivative by the limit of the fractional incremental ratio [20]

$$
D_{\theta}^{\alpha} f(z)=\mathrm{e}^{-\mathrm{i} \alpha \theta} \lim _{|h| \rightarrow 0} \frac{\sum_{j=0}^{\infty}(-1)^{j}\left(\begin{array}{c}
\alpha \\
j
\end{array}\right) f(z-j h)}{|h|^{\alpha}},
$$

where $h=\not \phi^{\mathrm{i} \theta}$ is a complex number, with $\theta \in(\pi, \pi]$. This expression can be interpreted as a generalization of the classical GL derivative.

We compute the derivative for two values of the parameter $\theta$, namely $\theta=0$ and $\theta=\frac{\pi}{2}$. Consequently, we have:

$$
D_{0}^{\alpha} f(z)=\lim _{|h| \rightarrow 0} \frac{\sum_{j=0}^{\infty}(-1)^{j}\left(\begin{array}{c}
\alpha \\
j
\end{array}\right) f(x-j|h|+\mathrm{i} y)}{|h|^{\alpha}},
$$

that we can consider as the partial derivative in order to $x$. We can write

$$
D_{0}^{\alpha}[u(x, y)+\mathrm{i} v(x, y)]=\frac{\partial^{\alpha} u(x, y)}{\partial x^{\alpha}}+\mathrm{i} \frac{\partial^{\alpha} v(x, y)}{\partial x^{\alpha}}
$$

Similarly for $\theta=\frac{\pi}{2}$

$$
\begin{array}{r}
D_{\frac{\pi}{2}}^{\alpha} f(z)=\mathrm{e}^{-\mathrm{i} \alpha \frac{\pi}{2}} \lim _{|h| \rightarrow 0} \frac{\sum_{j=0}^{\infty}(-1)^{\jmath}\left(\begin{array}{l}
\alpha \\
j
\end{array}\right) f(x+\mathrm{i}(y-j|h|))}{|h|^{\alpha}}, \\
D_{\frac{\pi}{2}}^{\alpha}[u(x, y)+\mathrm{i} v(x, y)]=\mathrm{e}^{-\mathrm{i} \alpha \frac{\pi}{2}}\left[\frac{\partial^{\alpha} u(x, y)}{\partial y^{\alpha}}+\mathrm{i} \frac{\partial^{\alpha} v(x, y)}{\partial y^{\alpha}}\right] \\
D_{\frac{\pi}{2}}^{\alpha}[u(x, y)+\mathrm{i} v(x, y)]=\left[\cos \left(\alpha \frac{\pi}{2}\right) \frac{\partial^{\alpha} u(x, y)}{\partial y^{\alpha}}+\sin \left(\alpha \frac{\pi}{2}\right) \frac{\partial^{\alpha} v(x, y)}{\partial y^{\alpha}}\right] \\
+\mathrm{i}\left[-\sin \left(\alpha \frac{\pi}{2}\right) \frac{\partial^{\alpha} u(x, y)}{\partial y^{\alpha}} \cos \left(\alpha \frac{\pi}{2}\right) \frac{\partial^{\alpha} v(x, y)}{\partial y^{\alpha}}\right]
\end{array}
$$


Equating the real and imaginary parts of both derivatives we obtain the fractional version of the Cauchy-Riemann conditions

$$
\begin{aligned}
& \frac{\partial^{\alpha} u(x, y)}{\partial x^{\alpha}}=\cos \left(\alpha \frac{\pi}{2}\right) \frac{\partial^{\alpha} u(x, y)}{\partial y^{\alpha}}+\sin \left(\alpha \frac{\pi}{2}\right) \frac{\partial^{\alpha} v(x, y)}{\partial y^{\alpha}} \\
& \frac{\partial^{\alpha} v(x, y)}{\partial x^{\alpha}}=-\sin \left(\alpha \frac{\pi}{2}\right) \frac{\partial^{\alpha} u(x, y)}{\partial y^{\alpha}}+\cos \left(\alpha \frac{\pi}{2}\right) \frac{\partial^{\alpha} v(x, y)}{\partial y^{\alpha}}
\end{aligned}
$$


With $a=1$ we obtain the classical Cauchy-Riemann equations

$$
\begin{aligned}
& \frac{\partial u(x, y)}{\partial x}=\frac{\partial v(x, y)}{\partial y} \\
& \frac{\partial v(x, y)}{\partial x}=-\frac{\partial u(x, y)}{\partial y}
\end{aligned}
$$

Furthermore, with $a=2$ we obtain two interesting relations

$$
\begin{aligned}
& \frac{\partial^{2} u(x, y)}{\partial x^{2}}+\frac{\partial^{2} u(x, y)}{\partial y^{2}}=0 \\
& \frac{\partial^{2} v(x, y)}{\partial x^{2}}+\frac{\partial^{2} v(x, y)}{\partial y^{2}}=0
\end{aligned}
$$

The expressions occur in the study of the harmonic functions.

With some manipulation of (6) and (21) it yields:

$$
\begin{aligned}
& \frac{\partial^{\alpha} u(x, y)}{\partial x^{\alpha}}+\frac{\partial^{\alpha} u(x, y)}{\partial y^{\alpha}}=\cot \left(\alpha \frac{\pi}{4}\right)\left[\frac{\partial^{\alpha} v(x, y)}{\partial x^{\alpha}}-\frac{\partial^{\alpha} v(x, y)}{\partial y^{\alpha}}\right] \\
& \frac{\partial^{\alpha} v(x, y)}{\partial x^{\alpha}}+\frac{\partial^{\alpha} v(x, y)}{\partial y^{\alpha}}=\cot \left(\alpha \frac{\pi}{4}\right)\left[\frac{\partial^{\alpha} u(x, y)}{\partial x^{\alpha}}-\frac{\partial^{\alpha} u(x, y)}{\partial y^{\alpha}}\right]
\end{aligned}
$$

We obtain expressions that can be considered as a generalization of the Laplacian for fractional orders. As it is obvious the corresponding Fourier transform becomes:

$$
\mathcal{F}\left[\frac{\partial^{\alpha} u(x, y)}{\partial x^{\alpha}}+\frac{\partial^{\alpha} u(x, y)}{\partial y^{\alpha}}\right](k)=\left[\left(\mathrm{i} k_{1}\right)^{\alpha}+\left(\mathrm{i} k_{2}\right)^{\alpha}\right] \hat{u}(\bar{k})
$$

with $a=2$ we recover the above result (8).

A similar procedure from equation (3), but with $\theta=\pi$ and $\theta=\frac{\pi}{2}$, leads to identical equations involving the backward derivatives.

For the Riesz solution of the 2D case and $a=1$ and $n=2$ we have a problem. If we compute the inverse of $|\vec{k}|^{2}=k^{2}+k^{2}$, then we obtain a sum of two second-order derivatives of the Dirac impulse in agreement with (24) and (19). However, if we try to use (14) the computation of the integral reveals problems.

\subsection{The $n D$ Laplacian}

We mentioned above that the Riesz potential implements a fractional negative power of the classical Laplacian. In particular it implements the inverses of the Laplacian and the biLaplacian. On the other hand, the Riesz derivative presented in (14), neither implements the integer order Laplacian, nor the bi-Laplacian [5].

A new fractional Laplacian obtained as the divergence of a gradient was obtained in [4]. Let us assume:

$$
\Delta^{\alpha} f(x)=D_{c x_{1}}^{2 \alpha_{1}}+D_{c x_{2}}^{2 \alpha_{2}}+\ldots+D_{c x_{n}}^{2 \alpha_{n}}
$$

where $\bar{a}=\left\{a_{1}, \cdots, a_{j}, \cdots, a_{n}\right\}$ and $D_{c x_{j}}^{2 a_{j}}, j=1,2, \ldots, n$, is the above centred derivative, valid for $a_{j}>-{ }_{2}, j=1,2, \ldots, n$. It is clear that this operator generalizes the classical 
one for $a_{j}=1, j=1,2, \ldots, n$, and has meaning for $a_{j}=\frac{1}{2}, j=1,2, \ldots, n$. Aside a sign it coincides with the 2D Laplacian we obtained previously. Attending to what we discussed previously about the 1D Riesz potential, we can extend the above formula for $a_{j}>\frac{1}{2}$, $j=1,2, \ldots, n$, substituting the centred derivatives by the 1D Riesz potential for each partial derivative

$$
\Delta^{\alpha} f(x)=\mathbb{I}_{R}^{2 \alpha_{1}} f\left(x_{1}, \ldots\right)+\mathbb{I}_{R}^{2 \alpha_{2}} f\left(\ldots, x_{2}, \ldots\right)+\ldots+\mathbb{I}_{R}^{2 \alpha_{n}} f\left(\ldots, x_{n}\right)
$$

These two last formulae can be considered as being complementary in the sense that (1) is valid for orders less than 1 , where the second becomes hyper-singular, while the second is regular for negative orders. In other words, (28) is valid for orders greater than- $\frac{1}{2}$ and (29) is valid for negative orders. Therefore, with this we obtain a Laplacian valid for any real order.

\subsection{Discussion}

After having studied several formulations involving the fractional Laplacian, this subsection discusses some afterthoughts that emerge. In this line of thought, we can outline the following topics:

- The Riesz Laplacian is the current most ubiquitous used operator, since it is a fractional power of the integer order Laplacian. In fact, it has been used to obtain the fractional form of classical differential equations, such as the Schrödinger equation (see, e.g. $[1,2,7,8,15,21,22]$ ). This formulation leads to interesting results mainly with stochastic inputs such as the Lévy flights [21-23]. However, most formulations are 1D in space and adopt a decomposition of the Riesz potential as a sum of a left and a right RL derivative [24]. We can find important cases (e.g. the fractional Maxwell equations) where such an approach is not appealing [15]. In this paper we try to give some insight into a formulation of the Laplacian suitable for dealing with equations where the Riesz approach is not the most straightforward.

- The studied Laplacians are long-range operators, meaning that they are defined on $\mathrm{R}^{n}$. This raises some problems in introducing boundary conditions. The classical methodology must be modified to adapt to long-range operators. This is an open problem and we believe that its solution may bypass the modification of the involved functions, not necessarily by means of the operators. Probably one should switch from using derivatives defined in subsets of $\mathrm{R}^{n}$, as in the case of the usual RL and Caputo derivatives, towards the GL, the Liouville or the centred derivative $[16,17]$.

- The computer implementation of the Laplacians can adopt the previously referred to derivatives. In particular, for incremental ratio derivatives, such as the GL, we only have to calculate the approximation. It is interesting to mention that we can reformulate the whole theory to get a purely discrete formulation as proposed in [25], but this topic requires further studies. 


\section{Conclusions}

This study developed a fractional version of the Laplacian operator. First was formulated the generalization problem and their possible solutions. We analysed particular cases, namely the 1D and 2D Laplacians. Also, fractional Cauchy-Riemann conditions were obtained. We concluded that the Riesz potential is useful in defining the negative order fractional Laplacian, by means of the repeated application of $1 \mathrm{D}$ operators.

\section{Acknowledgments}

This work was partially funded by National Funds through the Foundation for Science and Technology of Portugal, under the project PEst-OE/EEI/UI0066/2011.

\section{References}

[1] Chen W and Holm S 2004 Fractional Laplacian time-space models for linear and nonlinear lossy media exhibiting arbitrary frequency dependency J. Acoust. Soc. Am. 115 1424-30

[2] Caffarelli L and Silvestre L 2007 An extension problem related to the fractional Laplacian Commun. Partial Diff. Eqns 32 1245-60

[3] Ortigueira M D, Rivero M and Trujillo J J 2014 Introduction to fractional Grun̈wald-Letnikov vectorial operators: fundamental definitions Proc. 2014 Int. Conf. on Fractional Differentiation and Its Applications (Catania, Italy, 24-27 June 2014)

[4] Ortigueira M D, Rivero M and Trujillo J J 2014 Introduction to fractional Grun̈wald-Letnikov vectorial operators: the Laplacian Proc. 2014 Int. Conf. on Fractional Differentiation and Its Applications (Catania, Italy, 24-27 June 2014)

[5] Ortigueira M D, Rivero M and Trujillo J J 2014 From a generalized Helmholtz theorem to the fractional Maxwell equations J. Phys. A: Math. Theor. Submitted

[6] Carracedo C M and Alix M S 2001 The Theory of Fractional Powers of Operators (North-Holland Mathematics Studies vol 187) (Amsterdam: Elsevier)

[7] Samko S G, Kilbas A A and Marichev O I 1993 Fractional Integrals and Derivatives: Theory and Applications (Amsterdam: Gordon and Breach)

[8] Kilbas AA,Srivastava H M and Trujillo J J 2006 Theory and Applications of Fractional Differential Equations (North-Holland Mathematics Studies vol 204) (Amsterdam: Elsevier)

[9] Valério D, Trujillo J J, Rivero M, Machado J T and Baleanu D 2013 Fractional calculus: a survey of useful formulas Eur. Phys. J. Spec. Top. 222 1827-46

[10] Zhou Y 2014 Basic Theory of Fractional Differential Equations (Singapore: World Scientific)

[11] de Oliveira E C and Machado J T 2014 A review of definitions for fractional derivatives and integrals Math. Problems Eng. 20145

[12] Riesz M 1949 L'integral de Riemann-Liouville et le problème de Cauchy Acta Math. 81 1-222

[13] Feller W 1952 On a generalization of Marcel Riesz' potentials and the semigroups, generated by them Communications du seminaire mathematique de université de Lund vol 21 (Lund: Gleerup) pp 72-81

[14] Rivero M, Trujillo J J and Velasco M P 2014 Int. Conf. on Fractional Differentiation and Its Applications (Catania, Italy, 24-27 June 2014)

[15] Tarasov V E 2008 Fractional vector calculus and fractional Maxwell's equations Ann. Phys. 323 2756-78

[16] Ortigueira M D 2006 Riesz potential operators and inverses via fractional centred derivatives Int. J. Math. Math. Sci. 200612

[17] Ortigueira M D 2006 Fractional central differences and derivatives J. Vib. Control 14 1255-66

[18] Ortigueira M D 2011 Fractional Calculus for Scientists and Engineers (Lecture Notes in Electrical Engineering vol 84) (Berlin: Springer)

[19] Ortigueira M D 2006 A coherent approach to non integer order derivatives Signal Process. 86 2505-15 
[20] Ortigueira M D, Rodríguez-Germá L and Trujillo J J 2011 Complex Grünwald-Letnikov, Liouville, RiemannLiouville and Caputo derivatives for analytic functions Commun. Nonlinear Sci. Numer. Simul. $16 \quad 4174-82$

[21] Buldyrev S V, Gitterman M, Havlin S, Kazakov A Y, da Luz M G E, Raposo E P, Stanley H E and Viswanathan G M 2001 Properties of Lévy ights on an interval with absorbing boundaries Phys. A: Stat. Mech. Appl. 302 148-61

[22] Buldyrev S V, Havlin S, Kazakov A Y, da Luz M G E, Raposo E P, Stanley H E and Viswanathan G M 2001 Average time spent by Lévy flights and walks on an interval with absorbing boundaries Phys. Rev. E 64 041108

[23] Zoia A, Rosso A and Kardar M 2007 Fractional Laplacian in bounded domains Phys. Rev. E 76021116

[24] Saichev A I and Zaslavsky G M 1997 Fractional kinetic equations: solutions and applications Chaos 7 753-64

[25] Ortigueira M D, Coito F J V and Trujillo J J 2014 Discrete-time differential systems Signal Process. Online, doi: 10.1016/j.sigpro.2014.03.004 